Annuaire suisse de politique de développement

22-2 | 2003

Société de l'information et coopération internationale

\title{
La bataille des logiciels libres
}

\section{Bernard Lang}

\section{OpenEdition}

\section{Journals}

Édition électronique

URL : http://journals.openedition.org/aspd/587

DOI : 10.4000/aspd.587

ISSN : 1663-9669

\section{Éditeur}

Institut de hautes études internationales et du développement

\section{Édition imprimée}

Date de publication : 1 novembre 2003

Pagination : 195-199

ISSN : 1660-5934

\section{Référence électronique}

Bernard Lang, «La bataille des logiciels libres ». Annuaire suisse de politique de développement [En ligne], 22-2 | 2003, mis en ligne le 23 mars 2010, consulté le 07 septembre 2020. URL : http:// journals.openedition.org/aspd/587 ; DOI : https://doi.org/10.4000/aspd.587 


\section{La bataille des logiciels libres}

\section{Bernard Lang*}

a dématérialisation, sous forme de logiciels, des éléments critiques de la technologie fait du contrôle des logiciels un enjeu majeur de l'économie et du développement technique de la société de l'information. Cette transfor-

mation est une occasion exceptionnelle pour les pays en développement, car l'économie de l'immatériel est bien différente de l'économie du matériel et leur est bien plus accessible. Encore faut-il s'y adapter par des structures économiques et techniques appropriées. Les logiciels libres en sont un exemple.

Le développement des technologies de l'information s'accompagne dans de nombreuses industries d'une décomposition des produits et des activités entre une partie matérielle (adaptable/programmable) et une partie immatérielle (qui réalise la programmation et l'adaptation aux besoins spécifiques). Cela permet une banalisation et une standardisation des composantes matérielles indépendamment des usages finaux, avec pour effet d'augmenter la concurrence et de favoriser la production de masse. Ces deux facteurs entraînent une baisse importante des prix d'éléments difficiles à industrialiser dans la plupart des économies émergentes, parce cela nécessiterait des investissements et des infrastructures d'approvisionnement, de production et de distribution qui sont généralement hors de leur portée.

Cette baisse des prix d'importations souvent inévitables est d'autant plus la bienvenue qu'elle s'accompagne d'une plus grande pérennité des matériels dont les fonctionnalités, l'utilité, le caractère innovant et l'évolutivité résident de plus en plus dans les programmes informatiques qui les contrôlent. L'usage de ces matériels peut souvent être adapté et pérennisé par une simple reprogrammation de leurs logiciels.

\section{Une ressource clé pour les pays pauvres}

Le rôle des logiciels devient donc déterminant. Or, si l'économie matérielle est sévèrement contrainte par ses structures de coûts et d'organisation, l'économie des logiciels obéit à des lois bien moins contraignantes, qui les mettent naturellement à la portée de tous. Comme toutes les productions immatérielles, maintenant numérisées, les technologies logicielles peuvent être développées de façon collaborative sur Internet, sans infrastructure industrielle, puis reproduites et diffusées par l'Internet avec un coût marginal nul. Diffusion et usage peuvent dont être économiquement viables à prix nul si tel est le choix des auteurs.

* Directeur de recherche à l'INRIA (Institut national de recherche en informatique et en automatique), $<$ www.inria.fr>, e-mail <Bernard.Lang@inria.fr> ; vice-président de 1'AFUL (Association francophone des utilisateurs de Linux et des logiciels libres), <www.aful.org $>$. 
Dans ce contexte se confrontent deux tendances économiques:

$\square$ les logiciels dit «propriétaires », diffusés uniquement sous forme d'applications exécutables, avec des licences extrêmement contraignantes quant à leur usage, et avec interdiction (légale et technique) de procéder à quelque analyse, adaptation ou amélioration que ce soit;

$\square$ les logiciels dits «libres», sans contrainte d'utilisation, fournis avec leur code source (nécessaire à leur compréhension technique, leur évolution et leur entretien) et la possibilité - légale et technique - de les étudier, les transformer, les adapter et les redistribuer ${ }^{1}$. Car les auteurs de logiciels libres choisissent d'exercer leurs droits en mettant ces logiciels à la disposition du public ${ }^{2}$.

Un premier effet de cette situation est que les logiciels libres sont généralement disponibles quasi gratuitement, car toute personne qui en a copie peut les donner légalement. Les logiciels propriétaires (logiciels commerciaux usuels) doivent généralement être importés et payés en devises fortes. Les logiciels libres, utilisables sans payer de licence, sont donc une ressource particulièrement intéressante pour tous les pays, et particulièrement les moins riches. Encore faut-il qu'ils répondent aux besoins techniques.

\section{Les trois marchés du logiciel}

Il y a, pour simplifier, trois marchés du logiciel: les ordinateurs personnels (familiaux ou professionnels), les serveurs et les logiciels embarqués qui gèrent l'informatique interne à divers produits (téléphones, voitures, appareillages électroniques divers).

Pour les serveurs, la démonstration de l'emploi des logiciels libres n'est plus à faire: le système libre Linux représente près de $30 \%$ des systèmes d'exploitation sur les serveurs d'entreprise, et $62 \%$ des sites web dans le monde sont gérés par le logiciel libre Apache. C'est l'avenir.

En ce qui concerne les ordinateurs personnels, les logiciels libres sont moins utilisés, principalement en raison des habitudes des usagers, difficiles à changer. On constate néanmoins une évolution sensible, notamment en faveur de la bureautique OpenOffice.org disponible pour toutes les machines.

Moins connus du public, les logiciels embarqués forment le plus gros marché. Les logiciels libres y occupent une place importante, parfois à l'initiative de grands groupes industriels parce que, par l'établissement de standards publics, ils favorisent l'interopérabilité, et donc la coopération et la concurrence. L'avantage des logiciels libres dans ce contexte est qu'ils laissent à l'entreprise une maîtrise totale de ses ressources logicielles, hors des décisions arbitraires d'un fournisseur, mais

1 La mise à disposition du code source à seule fin d'étude, sans en autoriser la libre utilisation, a un effet opposé à celui du logiciel libre. En particulier, la programmation indépendante de logiciels similaires peut alors donner lieu à des poursuites judiciaires pour plagiat.

2 Free Software Foundation, GNU's Not Unix!, <www.fsf.org> ; Open Source Initiative (OSI), The Open Source Definition, <www.opensource.org/docs/definition.php>. 
sans pour autant lui en imposer le développement - auquel elle peut néanmoins contribuer - et sans payer de licence, ce qui améliore sa compétitivité.

La liberté d'emploi et de transformation des logiciels permise par les licences libres n'est pas un simple choix idéologique mais un outil qui permet à tous de contribuer librement à l'élaboration d'un patrimoine logiciel commun. Cela suscite un mode de développement calqué sur celui - éprouvé par les siècles - de la science ouverte. Comme la recherche scientifique, le développement libre mêle intimement la coopération entre les acteurs développeurs, la concurrence entre des approches diverses et, enfin, le contrôle et la reconnaissance par les pairs (développeurs et utilisateurs), qui jouent le même rôle incitatif et sélectif sur le marché de la connaissance que la concurrence et la sélection des marchés économiques traditionnels ${ }^{3}$.

\section{Avantages pour les pays du Sud}

Ce mode de production a des effets positifs sur la qualité, comme en attestent depuis huit ans des études empiriques ${ }^{4}$ dont les résultats ont précédé les nombreuses analyses qui expliquent ce succès ${ }^{5}$. La transparence du libre accès au code pour une large communauté permet une meilleure correction des erreurs ${ }^{6}$ et surtout l'élimination des pièges et composants espions trouvés trop souvent dans le logiciel propriétaire, chose particulièrement grave pour un composant essentiel des infrastructures informationnelles. En outre, le développement décentralisé en réseaux impose une structuration rigoureuse des logiciels et un strict respect des standards, tous deux facteurs de qualité, de maintenabilité, d'adaptabilité et surtout d'interopérabilité et de libre concurrence.

L'efficacité du modèle vient aussi de ce que ce sont les mêmes acteurs qui sont développeurs et utilisateurs, que ces acteurs soient des grands groupes industriels (SUN, IBM, HP, EDF), des PME (O'Reilly, Trolltech), des associations (Debian, KDE), des collectivité territoriales (Estramadure) et des administrations (Allemagne, France), ou des particuliers (R. Stallman, L. Torvalds). Les motivations économiques sont multiples, mais les principales sont la qualité technique, la réduction des coûts par mutualisation sans contrôle extérieur, une plus grande indépendance dans l'usage d'une ressource stratégique, et la promotion d'activités économiques connexes. D'où un intérêt mondial des instances politiques dans la promotion du logiciel libre ${ }^{7}$.

First Monday, Peer-reviewed Journal on the Internet, <www.firstmonday.org> (rechercher: Linux, OSS, open-source, free software, GNU, FSF).

4 Stephen Shankland (CNET News), Study Lauds Open-source Code Quality, 19 February 2003, <http://news.com.com/2102-1001-985221.html>.

Eric S. Raymond, The Cathedral and the Bazaar, 1997, <http://catb.org/ esr/writings/cathedral-bazaar>. Ibid.

GrULiC (Grupo de Usuarios de Linux de Córdoba), Proposal for the Use of Open Technologies in the Government/Proposición para el uso de Tecnologías Abiertas en el Estado, 03/02/03, <http://proposicion.org.ar> ; Sam Kritikos, The State of Open Source (SOS), 3 February 2003, <www.gnacademy.org/twiki/bin/view/SOS> ; Réponse du député péruvien Edgar Villanueva Nuñez à Microsoft (10 langues), 8 avril 2002, et autres documents, <www.pimiento.linux.com/peru2ms>. 
Pour les pays en développement, les logiciels libres présentent de nombreux avantages. Sur le plan économique, ils permettent des économies substantielles en devises fortes sur le coût des licences et sur les services, en déplaçant vers l'économie locale des activités qui étaient auparavant importées. Cela favorise l'indépendance technologique et le développement économique par la création d'une industrie locale de services d'adaptation aux besoins du pays. Plus généralement, la disponibilité des codes sources est une ressource éducative qui permet de développer des compétences techniques de même niveau que dans le reste du monde. Enfin, cette égalité d'accès et de formation permet aux techniciens et développeurs locaux de s'intégrer au tissu technologique mondial et d'y contribuer.

De plus en plus, les logiciels sont et seront le vecteur incontournable de la communication, de la connaissance et de la culture. C'est une chance dans la mesure où tous les pays sont à égalité devant les ressources immatérielles - pour autant qu'ils puissent les utiliser librement, en toute indépendance, en jouant sur la flexibilité naturelle des créations immatérielles pour les adapter à leurs besoins et à leur génie propres. C'est ce que permettent les logiciels libres pour ce qui concerne les médias et les supports informatiques. C'est aussi, plus généralement, ce que permettent les «contenus libres», c'est-à-dire les ressources intellectuelles - artistiques, éducatives, techniques ou scientifiques - laissées par leurs créateurs en usage libre pour tous. Logiciels et contenus libres promeuvent, dans un cadre naturel de coopération entre égaux, l'indépendance et la diversité culturelle: l'intégration sans l'aliénation.

\section{Menaces du brevetage}

Le développement des logiciels libres repose en particulier sur le droit d'auteur qui, grâce à des licences comme la GPL ${ }^{8}$, permet d'éviter l'appropriation par le secret. Le droit d'auteur est traditionnellement celui qui protège les créations immatérielles, dont le logiciel fait partie. C'est un droit gratuit, et donc bien adapté à des créations qui peuvent aussi être produites et diffusées sans but lucratif.

Cette situation est cependant remise en cause par divers groupes d'intérêt qui souhaitent rendre brevetables les techniques logicielles. Pour certains grands groupes d'édition de logiciels propriétaires, le brevet est une arme puissante contre les nouveaux entrants, entreprises ou pays. Dans une série de rapports, dits «de Halloween »", la société Microsoft donne sa vision des logiciels libres et son analyse de leur impact. Elle y considère la brevetabilité des logiciels comme un moyen efficace de contenir cette évolution. En effet, un processus de création et de diffusion non lucratif ne peut assumer les coûts de dépôt des brevets, ni celui des contentieux. Ceux-ci sont inévitables car, s'il est exceptionnel - et très rarement accidentel - d'être en contrefaçon pour le droit d'auteur, il est en revanche très courant d'être accidentellement et inconsciemment en contrefaçon de brevet, avec des conséquences financières qui peuvent être dramatiques.

GNU General Public Licence, Version 2, June 1991, <www.gnu.org/copyleft/gpl.html>.

The Halloween Documents, <www.opensource.org/halloween>. 
Le développement des logiciels libres est techniquement et économiquement efficace, et c'est une grande opportunité pour les pays du Sud - et même, bien que de façon moins critique, pour ceux du Nord. Mais en cette instance, comme en bien d'autres, les pays en développement risquent d'être les premières victimes des excès et des extensions incontrôlées et injustifiées de la propriété intellectuelle. A l'occasion des négociations internationales, notamment à l'OMC et à l'OMPI, il est indispensable qu'il y ait une forte mobilisation sur ces enjeux qui conditionneront la participation de tous à la société de l'information et sa réussite économique, sociale et culturelle. 